\title{
Evolvability and Neurodegenerative Disease: Antagonistic Pleiotropy Phenomena Derived from Amyloid Aggregates
}

\author{
Makoto Hashimoto $^{\mathrm{a}, *}$, Gilbert Ho ${ }^{\mathrm{b}}$, Yoshiki Takamatsu ${ }^{\mathrm{a}}$, Yuka Shimizu ${ }^{\mathrm{a}}$, Shuei Sugama ${ }^{\mathrm{c}}$, \\ Takato Takenouchi $^{\mathrm{d}}$, Masaaki Waragai ${ }^{\mathrm{a}}$ and Eliezer Masliah ${ }^{\mathrm{e}}$ \\ ${ }^{a}$ Tokyo Metropolitan Institute of Medical Science, Setagaya-ku, Tokyo, Japan \\ ${ }^{\mathrm{b}}$ PCND Neuroscience Research Institute, Poway, CA, USA \\ ${ }^{\mathrm{c}}$ Department of Physiology, Nippon Medical School, Tokyo, Japan \\ ${ }^{\mathrm{d}}$ Institute of Agrobiological Sciences, National Agriculture and Food Research Organization, \\ Tsukuba, Ibaraki, Japan \\ ${ }^{\mathrm{e}}$ Division of Neurosciences, National Institute on Aging, Bethesda, MD, USA
}

Accepted 28 May 2018

\begin{abstract}
At present, the precise physiological role of neurodegenerative disease-related amyloidogenic proteins (APs), including $\alpha$-synuclein in Parkinson's disease and $\beta$-amyloid in Alzheimer's disease, remains unclear. Because of similar adaptability of both human brain neurons and yeast cells to diverse environmental stressors, we previously proposed that the concept of evolvability in yeast prion could also be applied to APs in human brain. However, the mechanistic relevance of evolvability to neurodegenerative disorders is elusive. Therefore, our objective is to discuss our hypothesis that evolvability and neurodegenerative disease may represent a form of antagonistic pleiotropy derived from the aggregates of APs. Importantly, such a perspective may provide an outlook of the entire course of sporadic neurodegenerative diseases.
\end{abstract}

Keywords: Evolvability, amyloidogenic proteins (APs), neurodegenerative disease, antagonistic pleiotropy

The physiological functions of the amyloidogenic proteins (APs) from neurodegenerative diseases, such as $\alpha$-synuclein $(\alpha S)$ in Parkinson's disease (PD) and $\beta$-amyloid $(A \beta)$ in Alzheimer's disease (AD), is currently unknown [1]. In this regard, many of the previous studies have described that APs might be involved in the vesicle functions. For instance, $\alpha \mathrm{S}$ was shown to regulate the size of the presynaptic

\footnotetext{
${ }^{*}$ Correspondence to: Makoto Hashimoto, Tokyo Metropolitan Institute of Medical Sciences, 2-1-6 Kamikitazawa, Setagaya-ku, Tokyo 156-8506, Japan. Tel.: +81 36834 2354; Fax: +81 35316 3150; E-mail: hashimoto-mk@igakuken.or.jp.
}

vesicle pools in primary hippocampal neurons [2] and to promote SNARE-complex assembly [3]. Similarly, $A \beta$ has been characterized as a modulator of synaptic plasticity, which may be important in the regulation of learning and memory $[4,5]$. These findings are consistent with a notion that impairment of APs may underlie the pathogenesis of neurodegenerative diseases, but may not explain why APs are expressed ubiquitously in neuronal and non-neuronal tissues.

Evolvability of yeast prion represents an amyloid-based genetic switch that confers multiple 
phenotypes for yeast to cope with diverse stressors in the fluctuating environment [6]. Given that the human brain is exposed to multiple stressors, including hyperthermia, oxidative stress, kindling, physical stress and neurotoxicity, a similar concept of the evolvability could be applied to APs, including $\alpha S$ and $A \beta$ [1]. Briefly, the heterogeneity of the stress-induced aggregates of APs might confer a phenotypic diversification that may be adaptive against such diverse stresses, in essence a form of hormesis [1]. Subsequently, stress-induced APs might be transmitted from parents to offspring via the germ line, leading to a preconditioning of offspring [1]. In support of our hypothetical view, recent studies reveal that APs are composed of structurally distinct populations in neurodegenerative conditions such as PD and AD [7, 8]. Furthermore, accumulating evidence suggests that the transgenerational transmission that enables inheritance of the acquired characteristics to environmental stresses not only occurs in microorganisms but also in mammals [9, 10]. Moreover, it was recently shown that semen contained multiple types of amyloid fibrils in the absence of disease, although their biological roles are unknown [11]. Taken together, it is suggested that APs in human brain may also demonstrate evolvability, which is evolutionarily advantageous for the neuronal survival of offspring, although the transgenerational transmission of APs is still elusive.

In contrast to the obvious benefits of APs evolvability during reproductive stages, it has been well established that APs aggregation may play a central role in the pathogenesis of neurodegenerative disorders in aging [12]. Indeed, a number of studies have shown that prefibrillar aggregates, including oligomers and misfolded monomers, may be more neurotoxic than mature fibrils [13]. Thus, the processes of evolvability and neurodegeneration may both be attributable to APs aggregation, and furthermore, it is possible that the detrimental effects of APs aggregation in neurodegenerative disorders may not have been weeded out because of the biological benefits of evolvability in the earlier reproductive stage (Fig. 1). This is reminiscent of the antagonistic pleiotropy hypothesis proposed by G. C. Williams a half century ago, suggesting that certain genes whose functions are beneficial during reproductive stages may exert later adverse effects in aging (Fig. 1) [14]. Although there had been few clear-cut examples of candidate genes [15], a recent study revealed pleiotropic associations of allelic variants in a $2 q 22$ region with risks of major human diseases and mortality [16]. Given that the evolvability of APs protofibrils may act as epigenetic [1], it is predicted that the interaction of both genetics and epigenetics might be necessary to facilitate antagonistic pleiotropy in evolvability and neurodegeneration.

Our hypothesis may provide further insight into mechanisms of neurodegenerative disease that have been difficult to resolve by conventional theories of neurodegeneration. Recently, characterization of genetic mutations in PD, especially in terms of mitochondrial dysfunction and proteasomal neurotoxic activity, has greatly improved our understanding of the basis of familial PD [17]. Yet, the pathological mechanisms underlying sporadic PD, especially early disease pathogenesis, remain obscure. Our concept based on evolvability as a normal biological function during reproduction, postulates the preexistence of AP oligomers derived from parental brains which encode neuronal survival information passed to offspring [1]. To expand on this, during reproductive life stages, $\alpha \mathrm{S}$ aggregation might be tightly regulated as a function of mitochondrial activity and ubiquitin-proteasome system. In turn, during aging, neurodegeneration could result from mitochondrial and ubiquitin- proteasomal dysfunction resulting in $\alpha \mathrm{S}$ aggregation and neurotoxicity. Thus, in this instance, both evolvability and PD may represent a form of antagonistic pleiotropy manifested through aggregates of $\alpha \mathrm{S}$.

Our hypothesis may also account for the antagonistic pleiotropy of apolipoprotein $\mathrm{E}$ (ApoE), a major risk factor for $\mathrm{AD}$ [18]. The gene encoding for ApoE is polymorphic with three major alleles, $\epsilon 2, \epsilon 3$, and $\epsilon 4$, among which the $\epsilon 3$ allele is the most common with regard to the general population [19]. The presence of one or more $\epsilon 4$ alleles greatly increases risk of sporadic $\mathrm{AD}$, whereas $\epsilon 2$ allele reduces risk and delays disease onset [19]. Despite association of the $\varepsilon 4$ (ApoE4) allele with sporadic AD in aging, ApoE4 has persisted through evolution against the pressures of natural selection, indicating that ApoE4 might be biologically beneficial during reproduction. Provided that ApoE4 binds to $A \beta$ and stimulates fibrillization [20], one might speculate that the evolvability of $A \beta$ might be enhanced by ApoE4.

In addition, neuroinflammation may be regarded as another example of the antagonistic pleiotropy caused by APs. It has been well characterized that dysregulation of neuroinflammation by APs may result in production of high levels of pro-inflam- 


\section{Physiological (Reproduction)}

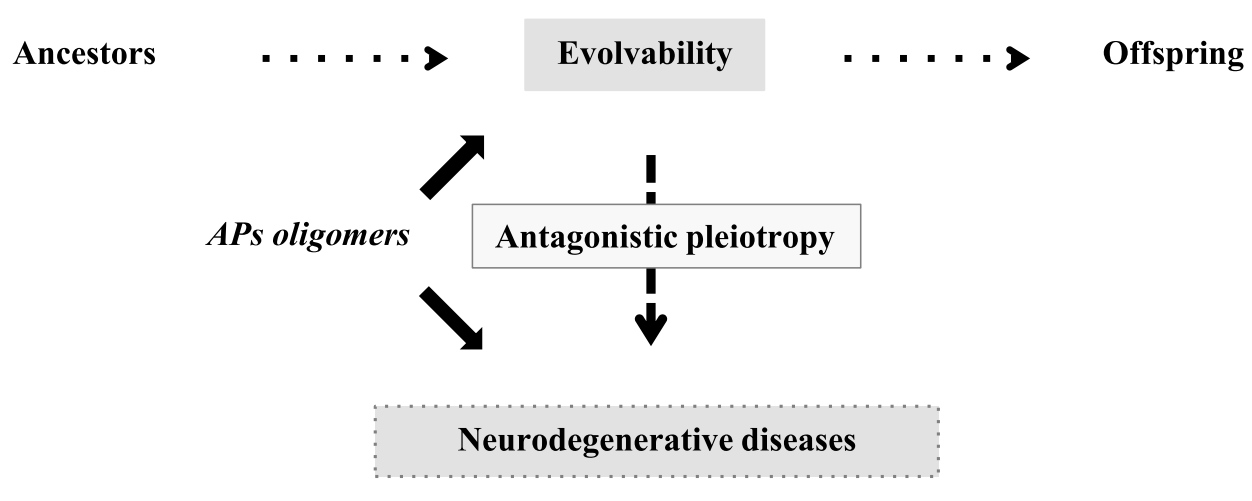

Pathological (Aging)

Fig. 1. Schematics of the pathophysiology of APs in human brain. Evolvability is supposed to be a physiological phenomenon during reproduction, whereas neurodegenerative diseases are pathological phenomena during the post-reproductive senescent period. Both are derived from the aggregates of APs and participate in an antagonistic pleiotropy relationship as illustrated.

matory and cytotoxic mediators, leading to exacerbation of neurodegenerative diseases, including PD and AD [21]. However, neuroinflammation is a doubleedged sword [22]. During the reproduction period in humans, neuroinflammation may be beneficial for the protection against various neuronal injuries, such as infection, toxic chemicals and physical insults [22].

Given the existence of prions in microorganisms such as yeast [6], evolvability may represent an ancient phenomenon in the course of evolution. In contrast, ample evidence suggests that neurodegenerative disease may be a relative latecomer in evolution. Although neurodegeneration is rapidly induced in the brains of spawning salmon [23], such a phenomenon may be a physiological strategy to supply nutrients to offspring. Furthermore, it is noteworthy that several neuropathologic studies in the brains of aged nonhuman primates, including chimpanzee and gorillas, demonstrated amyloid senile plaques and neurofibrillary tangles, although it is unclear whether such lesions are associated with a behavioral phenotype [24-26]. Notably, it has been shown that post-menopausal senescence of the animals may be affected by various factors, such as captivity and kinship dynamics [27]. Thus, it is possible that the presence of plaques and tangles in other primates might be an artificial phenomenon due to the prolonged length of post-menopausal senescence under the capturing conditions. In this context, neurodegenerative diseases might be associated with an extended postmenopausal lifespan, which is specific to human beings $[28,29]$. Taken together, we predict that the evolvability might be mechanistically situated upstream of neurodegenerative diseases and that their combined role in generating antagonistic pleiotropy may likely be a relatively recent phenomenon in human brain because of our extended postmenopausal lifespan.

In summary, evolvability of APs during reproduction may be biologically important and evolvability and aging-associated neurodegenerative diseases, such as $\mathrm{PD}$ and $\mathrm{AD}$, might be an antagonistic pleiotropy relationship mediated by aggregates of APs. Our view might provide a broader perspective with which to view the entire spectrum of sporadic neurodegenerative diseases, enabling possibility of identifying novel therapeutic strategies for such conditions. Certainly, continued investigation is warranted to strengthen this intriguing possibility.

\section{ACKNOWLEDGMENTS}

We are grateful for the continuous encouragement of Drs. Kaori Hashimoto (Tokyo Metropolitan Institute of Medical Science) and Maria del Carmen Ruiz de la Cruz (University of Chicago). 


\section{CONFLICT OF INTEREST}

The authors have no conflict of interest to report.

\section{REFERENCES}

[1] Hashimoto M, Ho G, Sugama S, Takamatasu Y, Shimizu Y, Takenouchi T, Waragai M, Masliah E (2018) Evolvability of amyloidogenic proteins in human brain. J Alzheimers Dis 62, 73-83.

[2] Murphy DD, Rueter SM, Trojanowski JQ, Lee VM (2000) Synucleins are developmentally expressed, and alphasynuclein regulates the size of the presynaptic vesicular pool in primary hippocampal neurons. J Neurosci 20, 3214-3220.

[3] Burre J, Sharma M, Tsetsenis T, Buchman V, Etherton MR, Sudhof TC (2010) Alpha-synuclein promotes SNAREcomplex assembly in vivo and in vitro. Science 329, 1663-1667.

[4] Pearson HA, Peers C (2006) Physiological roles for amyloid beta peptides. J Physiol 575, 5-10.

[5] Parihar MS, Brewer GJ (2010) Amyloid-beta as a modulator of synaptic plasticity. J Alzheimers Dis 22, 741-763.

[6] Wickner RB (2016) Yeast and fungal prions. Cold Spring Harb Perspect Biol 8, pii: a023531.

[7] Qiang W, Yau WM, Lu JX, Collinge J, Tycko R (2017) Structural variation in amyloid-beta fibrils from Alzheimer's disease clinical subtypes. Nature 541, 217-221.

[8] Theillet FX, Binolfi A, Bekei B, Martorana A, Rose HM, Stuiver M, Verzini S, Lorenz D, van Rossum M, Goldfarb D, Selenko P (2016) Structural disorder of monomeric alphasynuclein persists in mammalian cells. Nature 530, 45-50.

[9] Liu Y (2007) Like father like son. A fresh review of the inheritance of acquired characteristics. EMBO Rep $\mathbf{8}$, 798-803.

[10] Sharma A (2017) Transgenerational epigenetics: Integrating soma to germline communication with gametic inheritance. Mech Ageing Dev 163, 15-22.

[11] Roan NR, Sandi-Monroy N, Kohgadai N, Usmani SM, Hamil KG, Neidleman J, Montano M, Standker L, Rocker A, Cavrois M, Rosen J, Marson K, Smith JF, Pilcher CD, Gagsteiger F, Sakk O, O'Rand M, Lishko PV, Kirchhoff F, Munch J, Greene WC (2017) Semen amyloids participate in spermatozoa selection and clearance. Elife 6, e24888.

[12] Takamatsu Y, Ho G, Koike W, Sugama S, Takenouchi T, Waragai M, Wei J, Sekiyama K, Hashimoto M (2017) Combined immunotherapy with "anti-insulin resistance" therapy as a novel therapeutic strategy against neurodegenerative diseases. NPJ Parkinsons Dis 3, 4

[13] Sekiyama K, Takamatsu Y, Koike W, Waragai M, Takenouchi T, Sugama S, Hashimoto M (2016) Insight into the dissociation of behavior from histology in synucleinopathies and in related neurodegenerative diseases. J Alzheimers Dis 52, 831-841.
[14] Williams GC (1957) Pleiotropy, natural selection, and the evolution of senescence. Evolution 11, 398-411.

[15] Kirkwood TB (2005) Understanding the odd science of aging. Cell 120, 437-447.

[16] Kulminski AM, He L, Culminskaya I, Loika Y, Kernogitski Y, Arbeev KG, Loiko E, Arbeeva L, Bagley O, Duan M, Yashkin A, Fang F, Kovtun M, Ukraintseva SV, Wu D, Yashin AI (2016) Pleiotropic associations of allelic variants in a 2q22 region with risks of major human diseases and mortality. PLoS Genet 12, e1006314.

[17] Mizuno Y, Hattori N, Yoshino H, Hatano Y, Satoh K, Tomiyama H, Li Y (2006) Progress in familial Parkinson's disease. J Neural Transm Suppl, 191-204.

[18] Tuminello ER, Han SD (2011) The apolipoprotein e antagonistic pleiotropy hypothesis: Review and recommendations. Int J Alzheimers Dis 2011, 726197.

[19] Mahley RW (1988) Apolipoprotein E: Cholesterol transport protein with expanding role in cell biology. Science $\mathbf{2 4 0}$, 622-630.

[20] Ma J, Yee A, Brewer Jr HB, Das S, Potter H (1994) Amyloid-associated proteins alpha 1-antichymotrypsin and apolipoprotein E promote assembly of Alzheimer betaprotein into filaments. Nature 372, 92-94.

[21] Koenigsknecht-Talboo J, Landreth GE (2005) Microglial phagocytosis induced by fibrillar beta-amyloid and IgGs are differentially regulated by proinflammatory cytokines. J Neurosci 25, 8240-8249.

[22] Wyss-Coray T, Mucke L (2002) Inflammation in neurodegenerative disease-a double-edged sword. Neuron 35, 419-432.

[23] Maldonado TA, Jones RE, Norris DO (2000) Distribution of beta-amyloid and amyloid precursor protein in the brain of spawning (senescent) salmon: A natural, brain-aging model. Brain Res 858, 237-251.

[24] Rosen RF, Farberg AS, Gearing M, Dooyema J, Long PM, Anderson DC, Davis-Turak J, Coppola G, Geschwind DH, Pare JF, Duong TQ, Hopkins WD, Preuss TM, Walker LC (2008) Tauopathy with paired helical filaments in an aged chimpanzee. J Comp Neurol 509, 259-270.

[25] Perez SE, Raghanti MA, Hof PR, Kramer L, Ikonomovic MD, Lacor PN, Erwin JM, Sherwood CC, Mufson EJ (2013) Alzheimer's disease pathology in the neocortex and hippocampus of the western lowland gorilla (Gorilla gorilla gorilla). J Comp Neurol 521, 4318-4338.

[26] Cross R (2017) Elderly chimps may get Alzheimer's disease. Science 357, 440

[27] Croft DP, Brent LJ, Franks DW, Cant MA (2015) The evolution of prolonged life after reproduction. Trends Ecol Evol 30, 407-416.

[28] Rapoport SI (1989) Hypothesis: Alzheimer's disease is a phylogenetic disease. Med Hypotheses 29, 147-150.

[29] Finch CE, Austad SN (2015) Commentary: Is Alzheimer's disease uniquely human? Neurobiol Aging 36, 553-555. 